\title{
ELECTRONIC BAND STRUCTURE AND CALCULATED PHOTOEMISSION SPECTRA OF $\mathrm{USi}_{3}$ COMPOUND
}

\author{
J.A. Morkowski, A. Szajek and A. Jeziershi \\ Institute of Molecular Plysics, Polish Academy of Sciences \\ Smoluchowskiego 17, 60-179 Poznań, Poland
}

\begin{abstract}
The electronic band structure for $\mathrm{USi}_{3}$ was recalculated for varying ratios of the Wigner-Seitz radii, for $\mathrm{U}$ and $\mathrm{Si}$ atoms. The partial densities of states were used to calculate photoemission spectra which were compared with a vailable experimental clata.
\end{abstract}

PACS numbers: $71.20 .-b, 71.20 . G j, 79.60 .-\mathrm{i}$

\section{Introduction}

The isostructural series of compounds $\mathrm{UX}_{3}$ where $\mathrm{X}$ is a group-IV element $\mathrm{Si}$, $\mathrm{Ge}, \mathrm{Sn}$ or $\mathrm{Pb}$ provide interesting examples of diversified magnetic behaviour of actinide compounds in which $5 f$ electrons exhibit various degrees of localization due to the interplay of direct overlap of $5 f$ functions and the effects of hybridization. Some experimental data [1] indicate that $\mathrm{USi}_{3}$ behaves as a Pauli-type paramagnetic metal, $\mathrm{UGe}_{3}$ and $\mathrm{USn}_{3}$ can be considered as spin fluctuating systems whereas $\mathrm{UPb}_{3}$ should be well described by the band-structure theory based upon the local density approximation. The existing band structure data [2-4] differ in some details, particularly in the value of the density of states at the Fermi level. The aim of the present paper is to evaluate the band-structure results by comparing them with experimental data, mainly from photocmission spectra.

\section{Band structure}

The band structure was calculated by the tight binding linear muffin tin orbital method in the atomic sphere approximation (TB-LMTO-ASA) with the spin-orbit interactions taken into account for the experimental lattice constant $(a=4.0348(8) \AA)$. Details of the calculations and the total and partial densities of states (DOS) were reported recently [4]. A disturbing feature was that the calculated values of $\operatorname{DOS}\left(E_{\mathrm{F}}\right)$ were significantly different $(74,65.08$ and 44.56 states/(Ry cell) in Refs. [2, 3] and [4], respectively). The value of $(1+\lambda) \operatorname{DOS}\left(E_{\mathrm{F}}\right)=80$ states/(Ry cell) derived from the electronic specific heat data [5] although seems to favour rather the higher values of $\operatorname{DOS}\left(E_{\mathrm{F}}\right)$ is not 

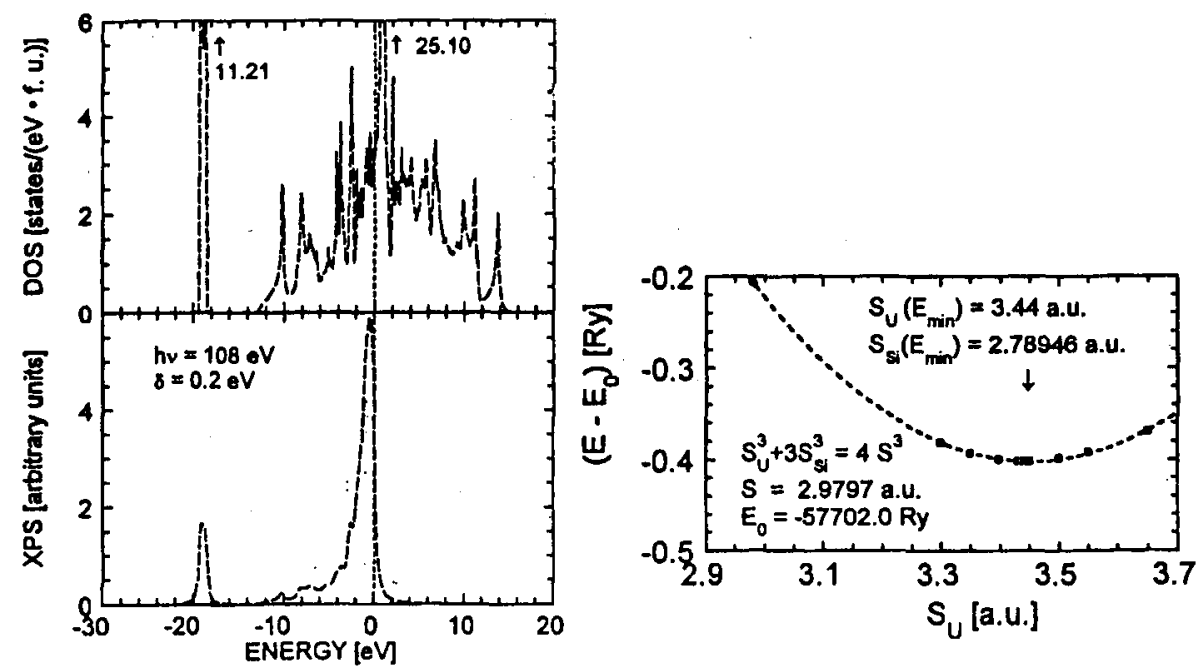

Fig. 1. Left panels: total $\operatorname{DOS}(E)$ for $\mathrm{USi}_{3}$ (for both spins, per formula unit) calculated for $S_{\mathrm{U}} / S_{\mathrm{Si}}$ corresponding to the minimum of the total energy and the photoemission spectrum. Right panel: dependence of the total electronic energy on the Wigner-Seitz radius for $\mathrm{U}$ atoms, $\mathrm{S}_{\mathrm{U}}$, calculated for fixed $S$ determined from the experimental value of the lattice constant.

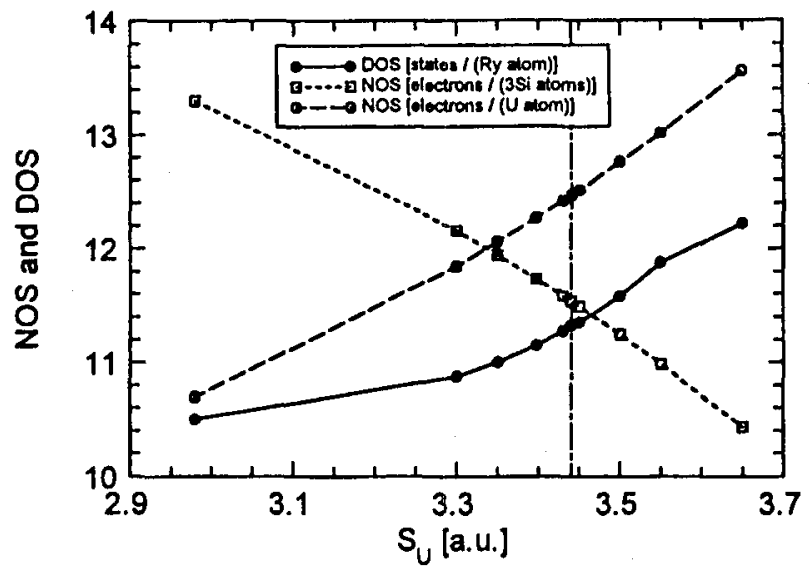

Fig. 2. Total $\operatorname{DOS}(E)$ (for both spins, per atom) vs. $S_{\mathrm{U}}$ (full line), and the number of states (NOS) inside the Wigner-Seitz spheres, $S_{U}$ around the $U$ atom (broken line) and $S_{\mathrm{Si}}$ around the Si atom (dotted line). The vertical line is at the value of $S_{\mathrm{U}}$ corresponding to the minimum of the total electronic energy. 
decisive since the phonon-enhancement factor is not known from independent experiments. The Pauli free-electron contribution to the paramagnetic susceptibility, proportional to $\operatorname{DOS}\left(E_{\mathrm{F}}\right)$ cannot be uniquely determined from experimental data because of the dominating orbital contribution (see e.g. [6]). In Refs. [2] and [3] the Wigner-Seitz radii for $U$ and $S i$ were assumed the same in spite of fact that atomic radii of $U$ and $S i$ differ substantially. In the TB-LMTO-ASA method [7] used in [3] and in the present calculations different Wigner-Seitz radii $S_{\mathrm{U}}$ and $S_{\mathrm{Si}}$, for $\mathrm{U}$ and $\mathrm{Si}$ atoms respectively, were taken. Calculating the total energy versus $S_{\mathrm{U}} / S_{\mathrm{Si}}$ a minimum is found for $S_{\mathrm{U}} / S_{\mathrm{Si}}=3.440 / 2.78946$ (Fig. 1, panel on the right). The average Wigner-Seitz radius $S$, defined by $4 S^{3}=S_{\mathrm{U}^{3}}+3 S_{\mathrm{Si}}^{3}$, was kept fixed and was determined from experimental lattice constant $a=4.0348(8) \AA[8]$ as $S=2.9797$ a.u. $\left((4 \pi / 3) S^{3}=a / 4\right.$ for the fcc unit cell). The total $\operatorname{DOS}\left(E_{\mathrm{F}}\right)$ increases with increasing $S_{\mathrm{U}}$ as it can be seen from Fig. 2 but does not come close to the values reported in [2] and [3]. The total $\operatorname{DOS}(E)$ for $S_{\mathrm{U}} / S_{\mathrm{Si}}$ corresponding to the minimum of energy is presented in Fig. 1 (upper left panel). The value of $\operatorname{DOS}\left(E_{F}\right)$ is 45.3 states/(Ry cell).

\section{Calculated plotoemission spectra}

To probe the occupied part of the electronic band structure the photoemission spectra (XPS) were calculated and compared with the existing experimental data. The XPS spectra were calculated from the partial DOS $(E)$ weighted with atomic photoemission cross-sections [9]. The finite experimental resolution is taken into account in calculations by convoluting the weighted $\operatorname{DOS}(E)$ with an energy dependent Lorentzian function parametrized by the IIWHM $=\delta$. An example of the XPS for photon energy $108 \mathrm{eV}$ and $\delta=0.2 \mathrm{eV}$ is presented in Fig. 1 (lower left panel). The peak at $E \approx-19 \mathrm{eV}$ below the Fermi energy $E_{\mathrm{F}}=0$ is due to $p$ electrons on $U$ atoms. The peak just below the Fermi level is dominated by $f$-electrons on U. In Fig. 3 calculated XPS curves for low binding energies are compared with
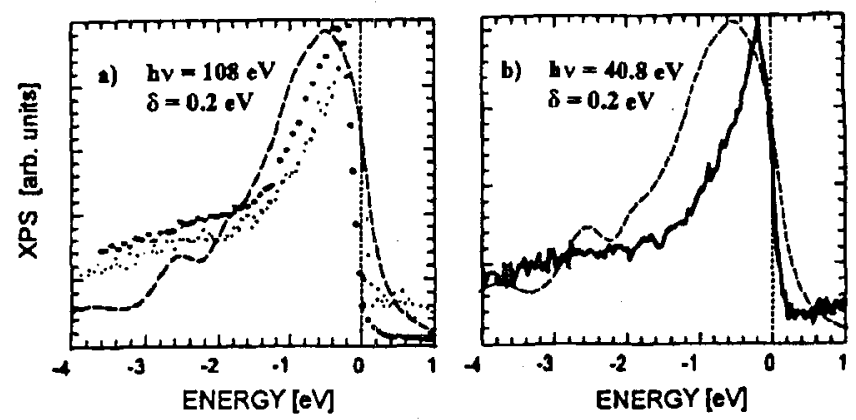

Fig. 3. The comparison of the calculated (dashed lines) and the experimental XPS spectra for the photon energies: (a) $108 \mathrm{eV}$, dots from [10], large circles from [11] and (b) $40.8 \mathrm{eV}$ [10]. The vertical line indicates the Fermi level. The bumps at $E \approx-2.4 \mathrm{eV}$ reveal contributions from the $p$ electrons from $\mathrm{Si}$ atoms. 
the published experimental data $[10,11]$. The calculated main peaks are shifted by about $0.2 \approx 0.3 \mathrm{eV}$ from the experimental ones, the bumps on the calculated plots, reflecting the contribution of $f$-electrons on $U$ atoms can be associated with features appearing in the experimental spectra.

\section{Acknowledgments}

This research was supported by the Committee for Scientific Research, grant No. 2 P302 00507.

\section{References}

[1] See e.g. W. Suski, R. Troć, in: Landoll-Börnstein, New Series Group III, Vol. 19, subvol. f2, Ed. H.P.J. Wijn, Springer-Verlag, Berlin 1993.

[2] A. Hasegawa, J. Magn. Magn. Maler. 52, 425 (1985).

[3] P. Strange, J. Phys. F, Met. Phys. 16, 1515 (1986).

[4] J.A. Morkowski, A. Szajek, Acta Phys. Slov. 46, 109 (1996).

[5] M.II. van Maaren, II.J. van Daal, K.II.J. Buschow, Solid State Commun. 14, 145 (1974).

[6] B. Nowak, O.J. Żogal, Physica B 215, 193 (1995).

[7] II.L. Skriver, The LMTO Melhod, Springer, New York 1984; J.P. Perdew, J.A. Chevary, S.II. Vosko, K.A. Jackson, M.R. Pederson, D.J. Singh, C. Fiolhais, Phys. Rev. B 46, 6671 (1992); G. Krier, O. Jepsen, A. Burkhardt, O.K. Andersen, The TB-LMTO-ASA Program, source code version 4.7, available upon request from the program authors.

[8] H.R. Ott, F. Hulliger, II. Rudigier, Phys. Rev. B 31, 1329 (1985).

[9] J.J. Yel, I. Lindau, At. Data Nucl. Dala Tables 32, 1 (1985).

[10] A.J. Arko, D.D. Koelling, C. Capasso, M. del Giudice, C.G. Olson, Phys. Rev. B 38, 1627 (1988).

[11] D.D. Sarma, F.U. Hillebrecht, C. Carbone, A. Zangwill, Phys. Rev. B 36, 2916 (1987). 